\title{
Combined ESPI and Digital Speckle Correlation technique for 3D displacement field measurement
}

\author{
Muravsky L.I., Sakharuk O.M., Kmet’ A.B., Voronyak T.I., Maksymenko O.P. \\ Karpenko Physico-Mechanical Institute of NAS of Ukraine \\ 5, Naukova str., Lviv 79601, Ukraine
}

System on the basis of combined ESPI and Digital Speckle Correlation technique for 3D displacement field measurement was created. This system allows measuring z-component by one step phase shift of reference wave on arbitrary angle and in-plane displacements by DSC technique. Experiments with steel beam possessing stress concentrator were carried out.

\section{Introduction}

It is well known that during surface deformation definition such interferometric techniques as Electronic Speckle-Pattern Interferometry (ESPI) which is widely used in experimental mechanics provide the most accuracy. Though possessing high spatial sensitivity this technique has limited range of measurement which does not exceed the average speckle size. During experimental determination of specimen deformations while loading one has motions for a value considerably bigger then acceptable measurement pitch and this do not allow us to conduct measurements of surface displacements or in better cases do not provide appropriate errors. At the same time, there is a number of means for surface displacement measurement based on Digital Speckle Correlation (DSC) technique and which have much bigger range of one step measurement. The range is determined by the dimensions of subimage. DSC techniques are more noiseless but their spatial resolution is less than for ESPI. DSC techniques do not have necessary spatial resolution for performing precise study for determination of initial dimension of material destruction zone. It is vivid that use of both these techniques during surface displacement measurement will let to obtain better results.

Andersson [1] has shown possibility to widen displacement measurement range by ESPI technique which is achieved by taking into consideration large displacement of specimen surface. To calculate displacement values at each image element of speckle-interferogram which need correction one measures displacements by DSC technique with the next interpolation of intermediate values for every image element. Proposed technique of range (for one step measurement) widen for ESPI can be used only for displacements and rotation of study specimen surface as a rigid body or in case of small surface deformations when we admit that deformations are homogeneous in the subimage limits.

Earlier Sjodahl [2] proposed to determine 3D surface displacements and deformations by combined technique when out-of-plane deformations were measured by phase-shifted interferometer and in-plane deformations - by DSC algorithm. Image (without reference wave) before and after specimen deformation, which is need to find in-plane deformations was received by combining images with different phase shifts. Spatial resolution of the setup in the $X Y$ plane was determined by subimage dimensions (32*32 pixels) and sensitivity was several percents of pixel dimensions and depend on optical system magnification. Measurement range along $Z$ coordinate was $5 \lambda$, sensitivity $-\lambda / 100$, and minimal spatial resolution was determined by pixel dimensions.

Advantages of ESPI and DSC combined use are obvious as DSC algorithm allows us to widen range of measured displacements by phase correction in ESPI, besides it is more stable for vibrations. From the other side use of ESPI for determination of in-plane displacements will be very effective as DSC sensitivity for out-of-plane displacements is very low.

\section{Theory}

While creating systems on the basis of combined ESPI and DSC technique one faces necessity to match these techniques spatial resolution and sensitivity in one measurement system. Thus surface displacement in the specimen plane on the value greater than average speckle dimension leads to considerable increase of speckle-image decorrelation. That's why there are rigid conditions for speckleimage formation with a set average speckle dimensions. Besides to determine in-plane displacements by DSC technique one needs to record a pair of contrast speckle images without reference wave for different loading steps. On the other hand, the measurement of out-of-plane surface displacement by phaseshifting ESPI methods demands recording as a rule of four speckle interferograms for each fixed surface 
location [2]. Such procedure is time-consuming and a mode of surface deformation during its fulfillment can be changed significantly. In order to avoid partially such lack, we have developed the technique of one step phase shift of reference wave on arbitrary angle [3]. The additional advantage of this technique is that the reference wave does not need to be shifted on the given angle. Proposed setup scheme for realization of combined technique is presented in Fig. 1.

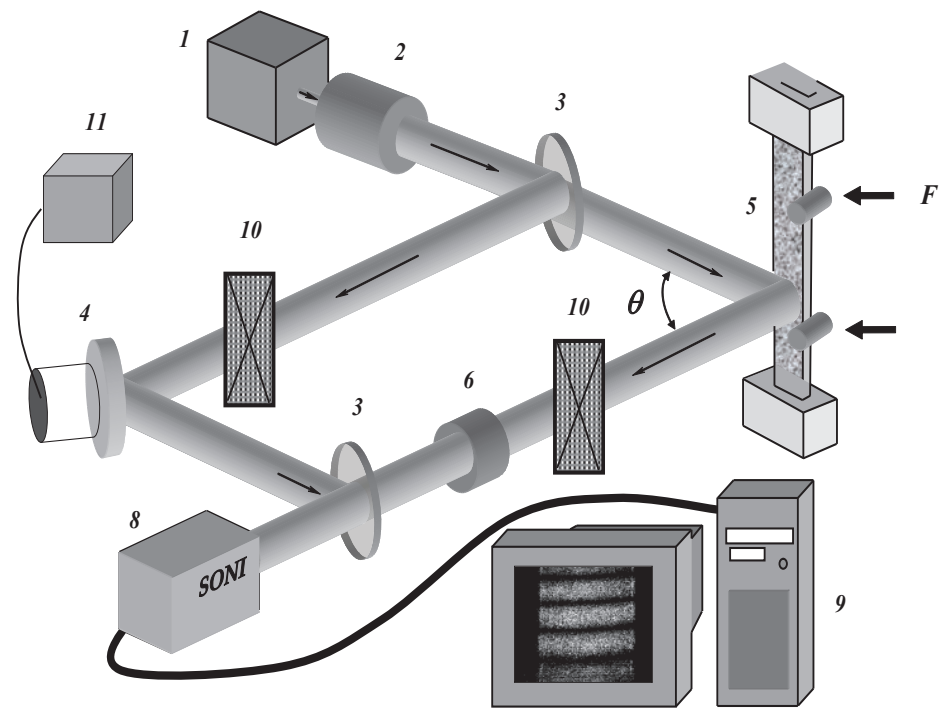

Fig. 1 - Setup scheme for combined use of ESPI and DSC techniques.

1 - laser; 2 - laser beam expander; 3 - beam splitter; 4 - phase shifting element;

5 - specimen with loading system; 6 - video camera lens; 7 - sweep mirror; 8 - video camera; 9 computer; 10 - electromechanical shutter; 11 - control unit for phase shifting element.

Algorithm for determine z-component by one step phase shift of reference wave on arbitrary angle consists in the next steps. One chooses ESPI phase-shifting technology which demands registration of seven speckle-images and single phase shift of reference wave on arbitrary angle $\alpha, 0<\alpha<\pi$.

Four speckle-images are recorded before specimen deformation

$$
\begin{array}{cc}
\mathrm{I}_{1}=\mathrm{I}_{\mathrm{Obj}}+\mathrm{I}_{\mathrm{Ref}}+\mathrm{I}_{\mathrm{m}} \cos \varphi, & \mathrm{I}_{2}=\mathrm{I}_{\text {Obj }}+\mathrm{I}_{\text {Ref }}+\mathrm{I}_{\mathrm{m}} \cos (\varphi+\alpha) \\
\mathrm{I}_{3}=\mathrm{I}_{\mathrm{Obj}}, & \mathrm{I}_{4}=\mathrm{I}_{\text {Ref }}
\end{array}
$$

and three after

$$
\begin{gathered}
\mathrm{I}_{5}=\mathrm{I}_{\mathrm{Obj}}^{\prime}+\mathrm{I}_{\mathrm{Ref}}+\mathrm{I}_{\mathrm{m}}^{\prime} \cos (\varphi+\Delta \varphi), \quad \mathrm{I}_{6}=\mathrm{I}_{\mathrm{Obj}}^{\prime}+\mathrm{I}_{\mathrm{Ref}}+\mathrm{I}_{\mathrm{m}}^{\prime} \cos (\varphi+\Delta \varphi+\alpha), \\
\mathrm{I}_{7}=\mathrm{I}_{\mathrm{Obj}}^{\prime}
\end{gathered}
$$

where $\mathrm{I}_{\mathrm{m}}=2 \sqrt{\mathrm{I}_{\text {Obj }} \mathrm{I}_{\text {Ref }}}, \mathrm{I}_{\mathrm{m}}^{\prime}=2 \sqrt{\mathrm{I}_{\text {Obj }}^{\prime} \mathrm{I}_{\text {Ref }}}$,

$\mathrm{I}_{i}$ - recorded value of $x, y$ pixel intensity in image $i, i=1,2,3,4,5,6,7$,

$\mathrm{I}_{3}=\mathrm{I}_{\mathrm{Obj}}$ i $\mathrm{I}_{7}=\mathrm{I}_{\mathrm{Obj}}^{\prime}$ - speckle-images with shut down reference wave,

$\mathrm{I}_{4}=\mathrm{I}_{\text {Ref }}-$ speckle-image with shut down object wave,

$\varphi$ - speckle random phase,

$\alpha$ - unknown angle of reference wave phase shift,

$\Delta \varphi$ - to be extracted phase information about out-of-plane surface displacements of specimen under loading.

In this case to determine $\Delta \varphi$ :

$$
\Delta \varphi=\operatorname{arctg}\left(\frac{\left(S_{1} C_{2}-C_{1} S_{2}\right) \sin \alpha}{C_{1} C_{2}+S_{1} S_{2}-\left(S_{1} C_{2}+C_{1} S_{2}\right) \cos \alpha}\right),
$$

where $C_{1}=\mathrm{I}_{1}-\mathrm{I}_{3}-\mathrm{I}_{4}, S_{1}=\mathrm{I}_{2}-\mathrm{I}_{3}-\mathrm{I}_{4}, C_{2}=\mathrm{I}_{5}-\mathrm{I}_{7}-\mathrm{I}_{4}$, a $S_{2}=\mathrm{I}_{6}-\mathrm{I}_{7}-\mathrm{I}_{4}$.

Let's show correctness of (3) equation. In agreement with equations (1) and (2):

$$
C_{1}=\mathrm{I}_{1}-\mathrm{I}_{3}-\mathrm{I}_{4}=\mathrm{I}_{\mathrm{m}} \cos \varphi \text {, }
$$




$$
\begin{aligned}
& S_{1}=\mathrm{I}_{2}-\mathrm{I}_{3}-\mathrm{I}_{4}=\mathrm{I}_{\mathrm{m}} \cos (\varphi+\alpha), \\
& C_{2}=\mathrm{I}_{5}-\mathrm{I}_{7}-\mathrm{I}_{4}=\mathrm{I}_{\mathrm{m}}^{\prime} \cos (\varphi+\Delta \varphi), \\
& S_{2}=\mathrm{I}_{6}-\mathrm{I}_{7}-\mathrm{I}_{4}=\mathrm{I}_{\mathrm{m}}^{\prime} \cos (\varphi+\Delta \varphi+\alpha) .
\end{aligned}
$$

Then

$$
\begin{gathered}
S_{1} C_{2}-C_{1} S_{2}=\mathrm{I}_{\mathrm{m}} \mathrm{I}_{\mathrm{m}}^{\prime}[\cos (\varphi+\alpha) \cos (\varphi+\Delta \varphi)-\cos \varphi \cos (\varphi+\Delta \varphi+\alpha)]= \\
=\frac{1}{2} \mathrm{I}_{\mathrm{m}} \mathrm{I}_{\mathrm{m}}^{\prime}\{[\cos (2 \varphi+\Delta \varphi+\alpha)+\cos (\Delta \varphi-\alpha)]-[\cos (2 \varphi+\Delta \varphi+\alpha)+\cos (\Delta \varphi+\alpha)]\}= \\
=\frac{1}{2} \mathrm{I}_{\mathrm{m}} \mathrm{I}_{\mathrm{m}}^{\prime}[\cos (\Delta \varphi-\alpha)-\cos (\Delta \varphi+\alpha)]=\mathrm{I}_{\mathrm{m}} \mathrm{I}_{\mathrm{m}}^{\prime} \sin \Delta \varphi \sin \alpha . \\
S_{1} C_{2}+C_{1} S_{2}=\mathrm{I}_{\mathrm{m}} \mathrm{I}_{\mathrm{m}}^{\prime}[\cos (\varphi+\alpha) \cos (\varphi+\Delta \varphi)+\cos \varphi \cos (\varphi+\Delta \varphi+\alpha)]= \\
=\frac{1}{2} \mathrm{I}_{\mathrm{m}} \mathrm{I}_{\mathrm{m}}^{\prime}\{[\cos (2 \varphi+\Delta \varphi+\alpha)+\cos (\Delta \varphi-\alpha)]+[\cos (2 \varphi+\Delta \varphi+\alpha)+\cos (\Delta \varphi+\alpha)]\}= \\
=\frac{1}{2} \mathrm{I}_{\mathrm{m}} \mathrm{I}_{\mathrm{m}}^{\prime}[2 \cos (2 \varphi+\Delta \varphi+\alpha)+\cos (\Delta \varphi-\alpha)+\cos (\Delta \varphi+\alpha)]= \\
=\mathrm{I}_{\mathrm{m}} \mathrm{I}_{\mathrm{m}}^{\prime} \cos (2 \varphi+\Delta \varphi+\alpha)+\mathrm{I}_{\mathrm{m}} \mathrm{I}_{\mathrm{m}}^{\prime} \cos \Delta \varphi \cos \alpha . \\
C_{1} C_{2}+S_{1} S_{2}=\mathrm{I}_{\mathrm{m}} \mathrm{I}_{\mathrm{m}}^{\prime}[\cos \varphi \cos (\varphi+\Delta \varphi)+\cos (\varphi+\alpha) \cos (\varphi+\Delta \varphi+\alpha)]= \\
=\frac{1}{2} \mathrm{I}_{\mathrm{m}} \mathrm{I}_{\mathrm{m}}^{\prime}[\cos (2 \varphi+\Delta \varphi)+\cos \Delta \varphi+\cos (2 \varphi+\Delta \varphi+2 \alpha)+\cos \Delta \varphi]= \\
=\mathrm{I}_{\mathrm{m}} \mathrm{I}_{\mathrm{m}}^{\prime}[\cos (2 \varphi+\Delta \varphi+\alpha) \cos \alpha+\cos \Delta \varphi]= \\
=\mathrm{I}_{\mathrm{m}} \mathrm{I}_{\mathrm{m}}^{\prime} \cos (2 \varphi+\Delta \varphi+\alpha) \cos \alpha+\mathrm{I}_{\mathrm{m}} \mathrm{I}_{\mathrm{m}}^{\prime} \cos \Delta \varphi .
\end{gathered}
$$

Relations (9) and (10) allow us to find $\mathrm{I}_{\mathrm{m}} \mathrm{I}_{\mathrm{m}}^{\prime} \cos (2 \varphi+\Delta \varphi+\alpha)$ in two ways. From the one side: $\mathrm{I}_{\mathrm{m}} \mathrm{I}_{\mathrm{m}}^{\prime} \cos (2 \varphi+\Delta \varphi+\alpha)=\frac{C_{1} C_{2}+S_{1} S_{2}-\mathrm{I}_{\mathrm{m}} \mathrm{I}_{\mathrm{m}}^{\prime} \cos \Delta \varphi}{\cos \alpha}$, and from the other:

$\mathrm{I}_{\mathrm{m}} \mathrm{I}_{\mathrm{m}}^{\prime} \cos (2 \varphi+\Delta \varphi+\alpha)=S_{1} C_{2}+C_{1} S_{2}-\mathrm{I}_{\mathrm{m}} \mathrm{I}_{\mathrm{m}}^{\prime} \cos \Delta \varphi \cos \alpha$. That's why we can proceed to next equation:

$$
\frac{C_{1} C_{2}+S_{1} S_{2}-\mathrm{I}_{\mathrm{m}} \mathrm{I}_{\mathrm{m}}^{\prime} \cos \Delta \varphi}{\cos \alpha}=S_{1} C_{2}+C_{1} S_{2}-\mathrm{I}_{\mathrm{m}} \mathrm{I}_{\mathrm{m}}^{\prime} \cos \Delta \varphi \cos \alpha
$$

Instead:

$C_{1} C_{2}+S_{1} S_{2}-\left(S_{1} C_{2}+C_{1} S_{2}\right) \cos \alpha=\mathrm{I}_{\mathrm{m}} \mathrm{I}_{\mathrm{m}}^{\prime} \cos \Delta \varphi\left(1-\cos ^{2} \alpha\right)=\mathrm{I}_{\mathrm{m}} \mathrm{I}_{\mathrm{m}}^{\prime} \cos \Delta \varphi \sin ^{2} \alpha$.

Having divided (8) by (12):

$$
\frac{S_{1} C_{2}-C_{1} S_{2}}{C_{1} C_{2}+S_{1} S_{2}-\left(S_{1} C_{2}+C_{1} S_{2}\right) \cos \alpha}=\frac{\mathrm{I}_{\mathrm{m}} \mathrm{I}_{\mathrm{m}}^{\prime} \sin \Delta \varphi \sin \alpha}{\mathrm{I}_{\mathrm{m}} \mathrm{I}_{\mathrm{m}}^{\prime} \cos \Delta \varphi \sin ^{2} \alpha}=\frac{\operatorname{tg} \Delta \varphi}{\sin \alpha} .
$$

From equation (13) will determine $\Delta \varphi=\operatorname{arctg}\left(\frac{\left(S_{1} C_{2}-C_{1} S_{2}\right) \sin \alpha}{C_{1} C_{2}+S_{1} S_{2}-\left(S_{1} C_{2}+C_{1} S_{2}\right) \cos \alpha}\right)$, what was necessary to prove.

Algorithm scheme is presented in Fig. 2.

To realize DSC algorithm, we record speckle-images of specimen surface in the initial state $\mathrm{I}_{3}=\mathrm{I}_{\mathrm{Obj}}$ and loaded state $\mathrm{I}_{7}=\mathrm{I}_{\mathrm{Obj}}^{\prime}$ after shut down of reference wave by electro-mechanic shutter. Received images were divided into subimages $h_{I 3}, h_{I 7}$ with dimensions $16 \times 16$ or $32 \times 32$ pixels and correlation of appropriate pairs of subimages was performed

$$
\widehat{C}(k, l)=\frac{1}{m} \sum_{i=0}^{m-1} \sum_{j=0}^{m-1}\left[h_{I 3}(i, j) \cdot h_{I 7}(i+k, j+l)\right],
$$

where $h_{I 3}$ - subimage from $I_{3} ; h_{I 7}$ - subimage from $I_{7} ; k, l=0,1 \ldots m-1$ - discrete values of correlation function. 


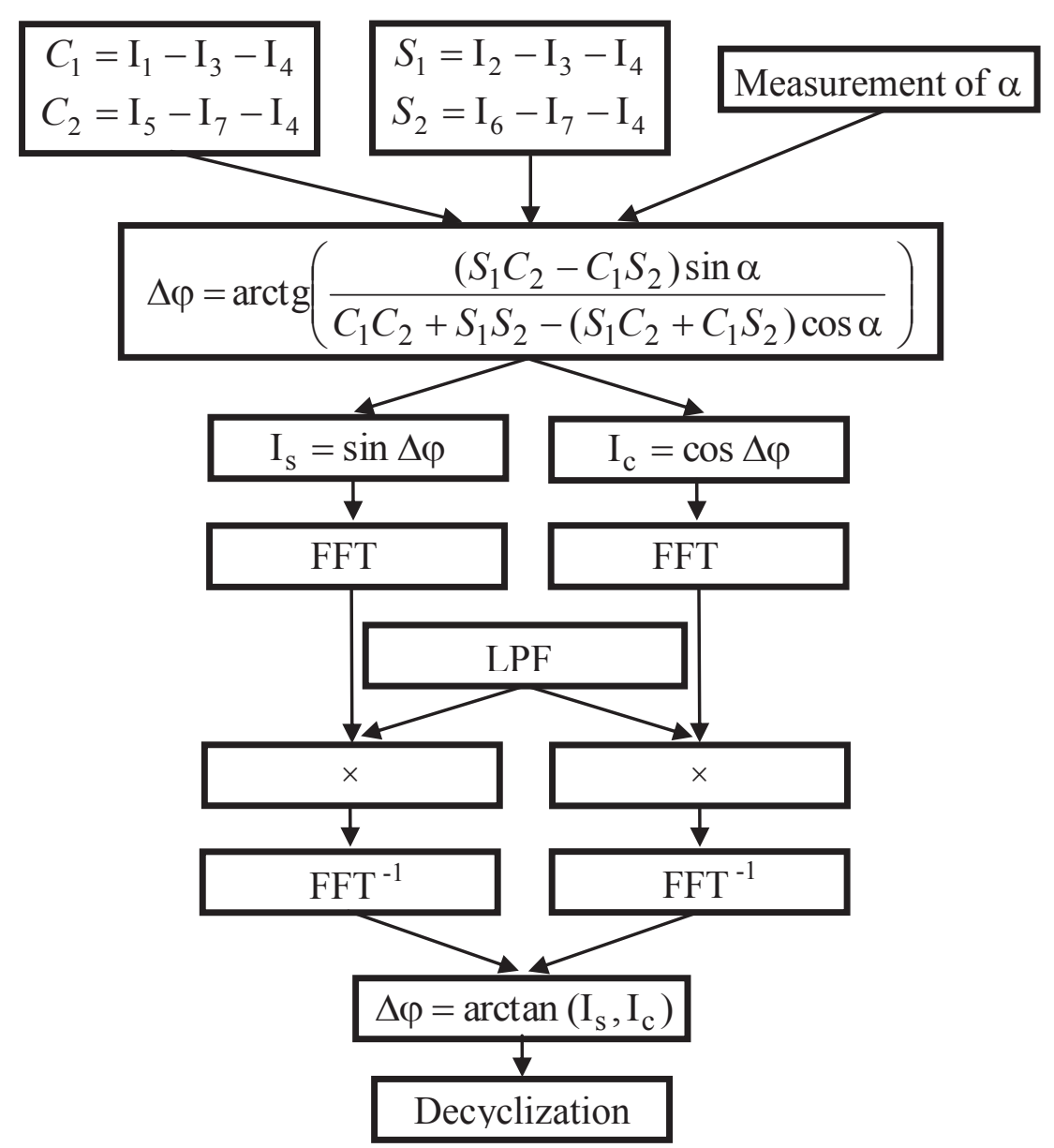

Fig. 2 - Flowgraph of algorithm for $z$-displacement definition

As direct calculation of correlation function (14) is rather time-consuming, we have used procedures of Fast Fourier Transform (FFT) [4]. Correlation function is

$$
\widehat{C}(k, l)=F^{-1}\left(H_{I 3} * H_{I 7}^{*}\right)
$$

where $H_{I 3}(r, s)=F\left(h_{I 3}\right) \mathrm{i} H_{I 7}^{*}(r, s)=F\left(h_{I 7}\right)$ - Fourier transform of the first and the second subimages; $F$ - symbol of FFT; $F^{1}$ - inverse FFT; * - complex conjugate function; $r, s=0,1 \ldots m-1$ subimage discrete spatial frequencies.

To increase ratio of correlation peak amplitude in comparison with surrounding noise one can use fraction power filter [5] to filter joint Fourier spectrum

$$
\hat{C}(k, l)=F^{-1}\left[H_{I 3} H^{*}{ }_{I 7} /\left|H_{I 3} H^{*}{ }_{I 7}\right|^{p}\right],
$$

where $0 \leq p \leq 1$. Choice of optimum parameter $p$ for this filter to minimize random errors of surface displacements was carried out by the technique developed by Muravsky et al. [6]

After processing every pair of speckle-images recorded without reference wave according to (16), one receive grate of correlation peaks whose displacement concerning subimage center characterize subimage displacement.

\section{Experiment}

The experimental setup was created according to the scheme shown in Fig. 1. Speckle-images were received by recording steel beam specimen (steel 45) surface. The specimen was subjected to loading equal to $200 \mathrm{~N}$ and was illuminated by He-Ne laser with $\lambda=632,8 \mathrm{~nm}$. Average speckle dimensions were 2 pixels. All speckle-images recorded by the digital camera SONY XCD-SX910CR $\left(1280 \times 960\right.$ pixels). We have studied specimen surface area of $14,7 \times 11 \mathrm{~mm}^{2}$ near stress concentrator. Recorded speckle-images were $800 \times 600$ pixels. The sensitivity of interferometer for z-displacement measurements was not worse than $10 \mathrm{~nm}$. To realize DSC algorithm, we record speckle-images of specimen surface in the initial state and loaded state after shut down of reference wave by electro- 
mechanic shutter. Received images were divided into subimages with dimensions $16 \times 16$ or $32 \times 32$ pixels and correlation of appropriate pairs of subimages was performed. In order to harmonize calculated $z$ (received by phase shifting interferometer) and $x y$ displacement (received by correlator) we have performed interpolation of $x y$ displacement results. The results of these displacement measurements are shown in Fig.3.

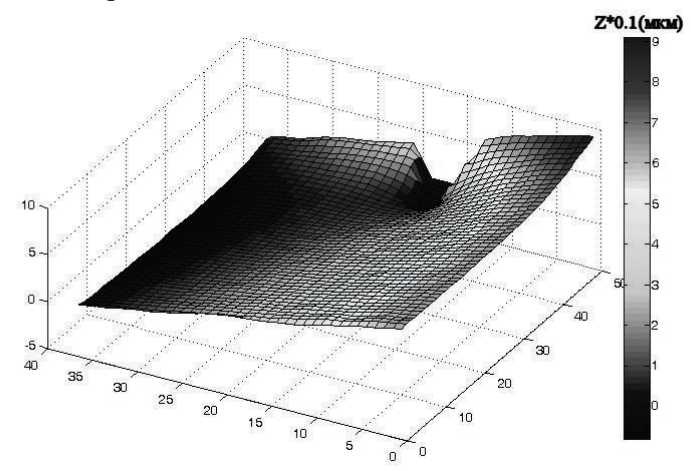

a)

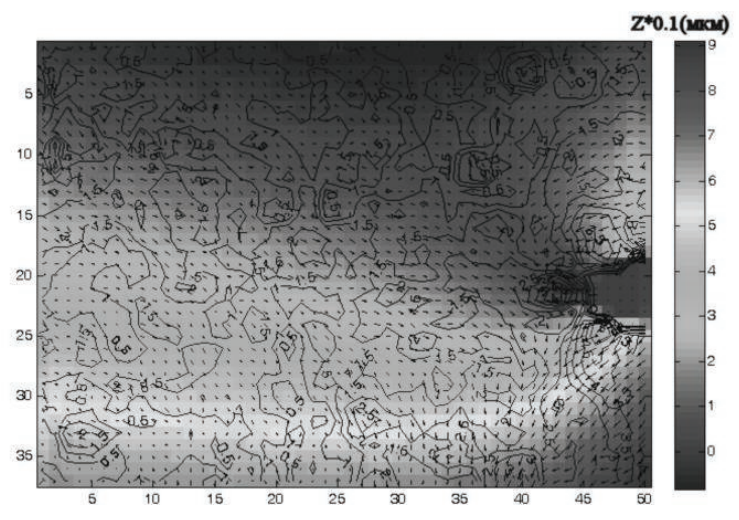

b)

Fig. 3. Received displacement field: a) $z$ displacements, b) $x y$ (vectors and isothetic) and $z$ (color) displacement.

\section{Conclusions}

We have shown advantages of ESPI and DSC combined use as DSC algorithm allow us to widen range of measured displacements by phase correction in ESPI. In comparison with well-known combined techniques [1,2], the proposed method uses the technique of one step phase shift of reference wave on arbitrary angle which is more convenient for studying the mechanical behavior of deformed specimens. From the other side, use of ESPI for determination of out-of-plane displacements will be very effective as DSC sensitivity for out-of-plane displacements is very low.

\section{References}

1. Andersson A., Runnemalm A., Sjodahl M. Digital speckle-pattern interferometry: fringe retrieval for large in-plane deformations with digital speckle photography/l Applied Optics. - September 1999. - Vol. 38. - N. 25. - P.5408-5412.

2. Siodahl M., Saldner H.O. Three-dimensional deformation field measurements with simultaneous TV holography and electronic speckle photography/l Applied Optics. - June 1997. - Vol. 36. - N. 1. - P.3645-3648.

3. Voronyak T.I., Kmet' A.B., Lychak O.V. A single-shot phase-shifting speckle interferometry // Physico-chemical mechanics of materials. - 2007.- Issue 4. - P. 93-102.

4. Chen D.J., Chiang F.P., Tan Y.S.and Don H.S. Digital speckle-displacement measurement using a complex spectrum method// Applied Optics - 1993. - Vol. 32. - N. 11. - P.1839-1849.

5. Vijaya Kumar B.V.K., Haseebrook L. Performance measures for correlation filters // Applied Optics - 1990. - Vol. 29. - N. 20. - P. 2997-3006.

6. Muravsky L.I., Polovynko T.I., Golynsky I.S.Criterion of fractional power filter optimization for digital speckle correlation // Information Extraction and Processing. - 2010. - Issue 33 (109). - P. 75-82. (in Ukrainian) 